\title{
List of Reviewers
}

\section{Volume 47, Issue 1}

Anne-Marie Emond

Bahar Haghighat

Boyd White

Cecile Badenhorst

Calla Coats

David Pariser

Felice Amato

Hsiao-Cheng Han

Linda Coles

Lorrie Blair

Marie-France Berard

Michelle Wiebe

Mindy R. Carter

Natalie LeBlanc

Patti Pente

Peter Gouzouasis 